\title{
Thirteen Drift Bands in PSR B0826-34
}

\author{
A. Esamdin \\ National Astronomical Observatories/Urumqi Observatory, CAS \\ Urumqi 830011, P. R. China
}

\author{
A. G. Lyne, M. Kramer, F. Graham-Smith \\ University of Manchester, Jodrell Bank Observatory, Macclesfield, \\ Cheshire SK11 9DL, UK \\ R. N. Manchester \\ Australia Telescope National Facility, CSIRO, P.O. Box 76, Epping \\ NSW 1710, Australia
}

\begin{abstract}
By developing a new technique, 13 drifting subpulses have been revealed across one period in PSR B0826-34. The variation of drift rate when drifting subpulses drift through different longitude regions are noticed. A weak emission mode was detected during its apparent null.
\end{abstract}

\section{Introduction}

PSR B0826-34 is known as a pulsar whose emission extends through the whole pulse period $(P=1.848 \mathrm{sec})$. The drift rate of its drifting subpulses shows wide variations include sign reversals. Durdin et al. (1979) noted that the pulsar exhibited nulls for 70 per cent of the time. Further investigations of this pulsar by Biggs et al. (1985) at $408 \mathrm{MHz}$ and $645 \mathrm{MHz}$ found emission at all longitudes, implying that this pulsar is an almost aligned rotator viewed from near the rotation axis. They found 5 to 6 bands of drifting subpulses, but were unable to trace these through the whole longitude range.

We have recorded sequences of individual pulses from PSR B0826-34, to investigate drifting subpulses round a complete track in the emitting region. We also found a weak integrated profile mode at $1374 \mathrm{MHz}$ during its apparent nulls.

\section{Observations and Data}

The single-pulse observations used in our analysis were made with the 64-m Parkes radio telescope using the central beam of the multibeam receiver system at $1374 \mathrm{MHz}$. Two high quality single-pulse sequences were obtained, on 2002 Sep 10 and Nov 1. The first sequence comprised 11670 individual pulses, with about 5900 continuously in strong emission mode; the second comprised 7530 individual pulses, with about 3900 continuously in the strong emission mode. 


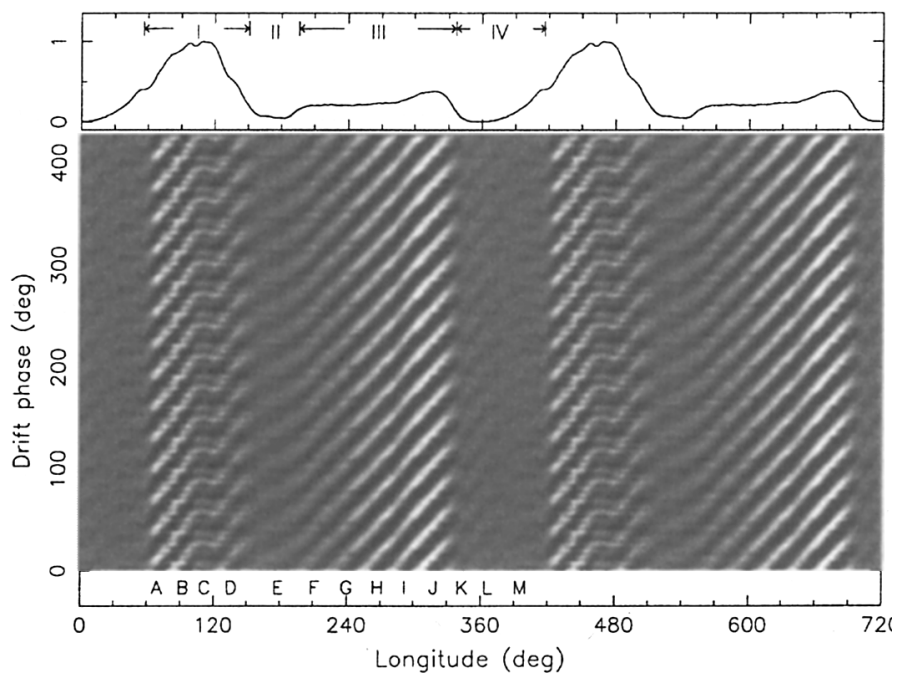

Figure 1. Integrated drifting-subpulse diagram in gray scale (lower panel). 13 integrated drifting subpulses are labeled from A to M. The upper panel shows integrated pulse profile. Four longitude regions (I, II, III and IV) are divided according to emission and drifting properties.

\section{Drifting Subpulses}

The integrated pulse profile at $1374 \mathrm{MHz}$ is shown in the upper panel of Figure 1. We need to integrate a long sequence of subpulses so as to follow more clearly the drifting pattern in weak emission regions II and IV. This is achieved by following the drift pattern and adding individual pulses grouped according to their drift phase. The drift phase in each separate pulse of the whole series was found by convolving the longitude region $190^{\circ}<l<325^{\circ}$ with a template.

We divided the average band interval in region III in 10 , and the pulses whose phases were located in the same phase intervals were co-added; the 10 profiles were then subtracted from the integrated pulse profile to give 10 remnant profiles. In the lower panel in Figure 1 we present the 10 remnant profiles in gray scale by repeating them twice in longitude and 14 times in drift phase.

The subpulses can now be seen through the whole range of longitude. 13 drifting subpulses are seen, labeled $A$ to $M$ in Figure 1. The changing inclination of the dark strips in the diagram indicates the variation of drift rate when drifting subpulses drift through a different longitude region.

\section{References}

Biggs, J. D., McCulloch, P. M., Hamilton, P. A., Manchester, R. N., \& Lyne, A. G. 1985, MNRAS, 215, 281

Durdin, J. M., Large, M. I., Little, A. G., Manchester, R. N., Lyne, A. G., \& Taylor, J. H. 1979, MNRAS, 186, 39P 